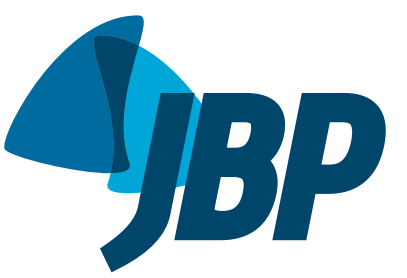

\title{
Liquid silicone injection in the chest wall simulating cysticercosis
}

\author{
Luiz Felipe Nobre1, Gláucia Zanetti², Edson Marchiori²
}

A 37-year-old homosexual Brazilian man presented with fever, chest pain, and several small, palpable lumps in the anterior chest wall. Physical examination revealed numerous mobile subcutaneous and intramuscular nodules measuring 1-2 cm in the anterior chest wall, with signs of a local inflammatory process. Chest CT scanning showed numerous round and oval nodules, most of which had rim calcifications, in the anterior chest wall. The nodules were apparently related to the muscles of the region (Figure 1 ).

Since the patient was from a cysticercosis endemic region, this infection was initially suspected. However, the CT findings were not suggestive of cysticercosis. The characteristic morphology of the calcifications observed in cysticercosis is "rice like" (or "cigar shaped"), and the calcifications are orientated along the long axes of the muscles. In addition, nodules in cysticercosis are distributed diffusely throughout the muscles. ${ }^{(1)}$ In our case, the calcifications presented with a rimmed aspect and occurred only in the anterior chest wall. Upon further discussion, the patient reported that he had undergone liquid silicone injection into the breasts, performed by an unskilled individual for soft-tissue augmentation, 12 years previously. In conclusion, sequelae of liquid silicone injection should be included in the differential diagnosis of chest wall calcifications.

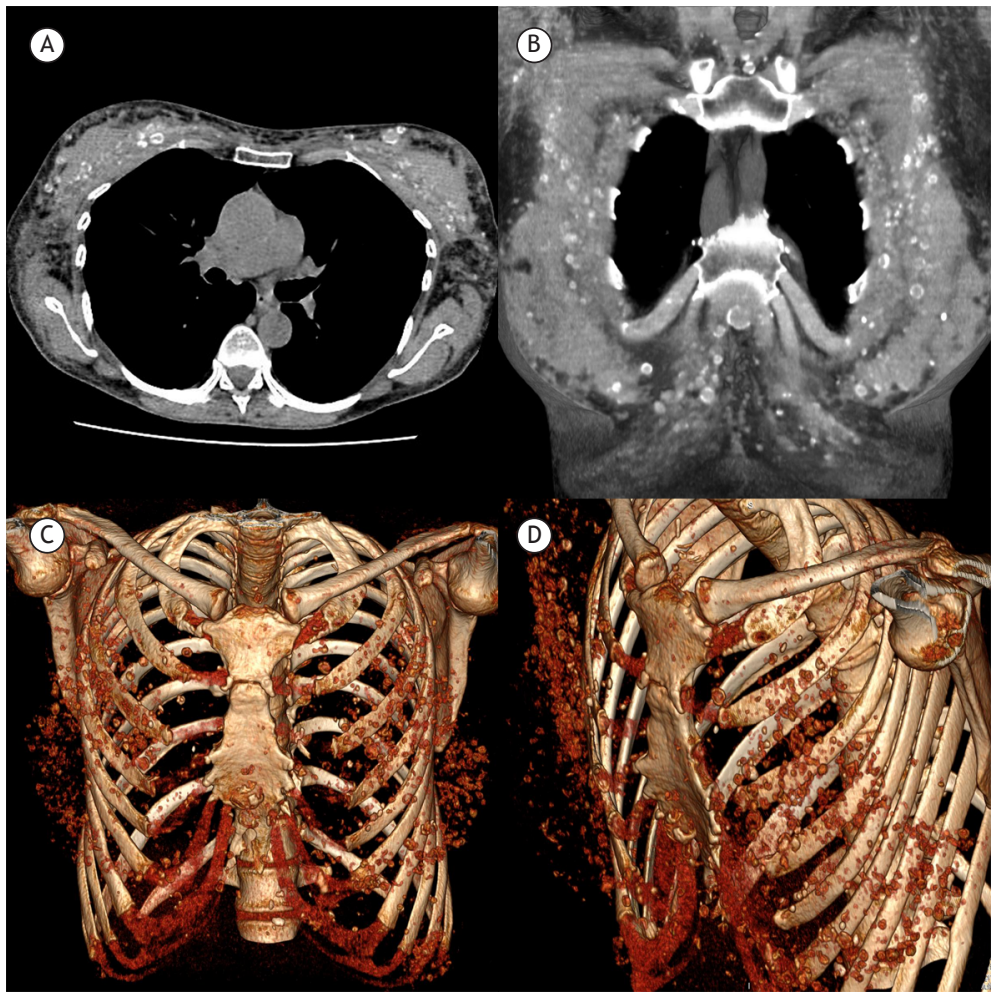

Figure 1. Axial (in A) and coronal (in B) CT reconstruction scans showing various bilateral round and oval nodules with rim calcifications in the anterior chest wall. Three-dimensional coronal (in C) and oblique (in D) CT reconstruction images demonstrated that the nodules were apparently related to the muscles of the region.

\section{REFERENCE}

1. Liu H, Juan YH, Wang W, Liang C, Zhou H, Ghonge NP, et al. Intramuscular cysticercosis: starry sky appearance. QJM. 2014;107(6):459-61. https://doi. org/10.1093/qjmed/hct243 